\title{
Recent Science from Australian Large-Scale Millimetre Mapping Projects: Proceedings from a Swinburne University Workshop
}

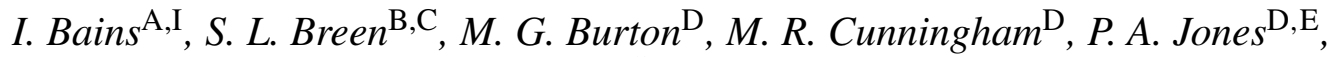

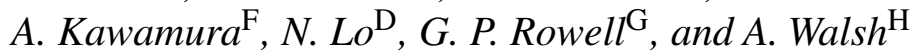 \\ A Centre for Astrophysics \& Supercomputing, Swinburne University of Technology, Hawthorn, VIC 3122 \\ ${ }^{B}$ School of Mathematics and Physics, University of Tasmania, Private Bag 37, Hobart, TAS 7001 \\ ${ }^{C}$ Australia Telescope National Facility, CSIRO, PO Box 76, Epping, NSW 1710 \\ D School of Physics, University of New South Wales, Sydney, NSW 2052 \\ E Departamento de Astronomía, Universidad de Chile, Casilla 36-D, Santiago, Chile \\ F Department of Astrophysics, Nagoya University, Furocho, Chikusaku, Nagoya 464-8602, Japan \\ G School of Chemistry \& Physics, University of Adelaide, Adelaide, SA 5005 \\ ${ }^{\mathrm{H}}$ Centre for Astronomy, James Cook University, Townsville, QLD 4811 \\ I Corresponding author. Email: ibains@astro.swin.edu.au
}

Received 2008 October 20, accepted 2009 February 20

\begin{abstract}
Since the recent upgrades to the Australia Telescope National Facility (ATNF) Mopra telescope back-end and receiver system, it has risen from an under-subscribed facility to a sought-after instrument with heavy international competition to gain time. Furthermore, the introduction of the on-the-fly mapping capability in 2004 has made this technique one of Mopra's most popular observing modes. In addition, the recent upgrade of the NANTEN millimetre-wavelength telescope to the sub-millimetre NANTEN2 instrument, has provided a complementary, higher-frequency facility to Mopra. A two-day workshop was held at Swinburne University in June 2008 to disseminate the current state of ongoing large-scale mapping projects and associated spin-offs that the telescopes' upgrades have facilitated, and to decide upon future research directions. Here, we provide a summary of the result-oriented talks as a record of the state of Australian-access single-dish millimetre science in 2008.
\end{abstract}

Keywords: acceleration of particles — ISM: clouds — ISM: jets and outflows — ISM: molecules — radio continuum: ISM — stars: formation

\section{Introduction}

As momentum builds towards the introduction of ALMA (the Atacama Large Millimetre Array), the ATNF Mopra ${ }^{1}$ telescope - the largest single-dish millimetre-wavelength facility in the southern hemisphere - has become a key instrument for precursor studies to some of the science planned for the Llano de Chajnantor array. Facilitating this rise in popularity has been a series of upgrades to Mopra's instrumentation. This began in 1999 with the extension of the solid surface of the dish to $22 \mathrm{~m}$ from the original $15 \mathrm{~m}$, followed by a series of software and computer upgrades, the introduction of on-the-fly (OTF) mapping in 2004, the installation of MMIC (Monolithic Microwave Integrated Circuit) receivers for $3 \mathrm{~mm}$ in 2005, $12 \mathrm{~mm}$ in 2006 and $7 \mathrm{~mm}$ in 2008, as well as, in 2006, the introduction of the 64000 -channel, 8-GHz bandwidth UNSW-MOPS (MOPra Spectrometer) digital filter bank

\footnotetext{
${ }^{1}$ The Mopra telescope is part of the Australia Telescope which is funded by the Commonwealth of Australia for operation as a National Facility managed by CSIRO. See http://www.narrabri. atnf.csiro.au/mopra/.
}

back-end ${ }^{2}$. The latter was aided by an ARC LIEF (Australian Research Council Linkage Infrastructure, Equipment and Facilities) grant to the Universities of New South Wales, Sydney and Monash and the CSIRO-ATNF.

As one of the strengths of single-dish telescopes lies in their large-scale mapping capability, the introduction of OTF saw a surge in the use of the Mopra telescope for this very technique. In particular, the star formation community seized upon the opportunity to map molecular line emission from molecular cloud complexes in the southern sky, which has resulted in many large-scale mapping projects, viz. the Delta Quadrant Survey (DQS; Bains et al. 2006; Wong et al. 2008; Lo et al. 2007, 2009), the Central Molecular Zone survey (CMZ; Jones et al. 2008), the $\mathrm{H}_{2} \mathrm{O}$ Southern Galactic Plane Survey (HOPS; Walsh et al. 2008) and the Magellanic Mopra Assessment (MAGMA; Ott et al. 2008), the first three of which are detailed in this paper. Table 1 gives the observing parameters of these surveys, along with those of the NANTEN telescope surveys also detailed in this publication. Note that MAGMA is a

\footnotetext{
${ }^{2}$ See http://www.narrabri.atnf.csiro.au/mopra/ obsinfo2.html for details of this system.
} 
${ }^{12} \mathrm{CO}$ and ${ }^{13} \mathrm{CO}$ targeted survey, not a map of a continuous region. The targets are the 114 brightest $\mathrm{CO}$ clouds from the NANTEN Large Magellanic Cloud (LMC) survey, in addition to $\sim 9$ clouds in the Small Magellanic Cloud (SMC).

The NANTEN2 ${ }^{3}$ single-dish telescope, situated in Chile, in many ways provides a complementary facility to Mopra, with its higher operating frequencies being suited to tracing the higher $J$ molecular transitions (whereas Mopra's strength is in observations of lower $J$ transitions). The strong collaborative link between UNSW, University of Sydney, Macquarie University and the NANTEN2 international university consortium (led by the University of Nagoya in Japan and the University of Cologne in Germany), aided by an ARC LIEF grant has permitted Australian access to this facility.

In June 2008 we deemed it timely to convene a twoday meeting at Swinburne University of Technology in Melbourne for Australian millimetre researchers and their overseas collaborators, in particular to disseminate the results of the recent large-scale mapping projects facilitated by the Mopra upgrades and NANTEN2 collaborative links, and to decide upon future research directions. Here, we present a summary of the result-orientated presentations from the meeting.

The outline of the paper is as follows. In Section 2, Andrew Walsh presents the first results from the $\mathrm{H}_{2} \mathrm{O}$ Southern Galactic Plane Survey. Michael Burton reports on preliminary results from the Central Molecular Zone mapping project in Section 3 and, in Section 4, Akiko Kawamura presents a large-scale molecular mapping programme of the Magellanic Clouds. In Section 5, Gavin Rowell details how the spatial correlation between $\gamma$ ray emission and that from molecular gas can reveal the origin of cosmic-ray hadrons. Section 6 is based on the UNSWled multi-molecular line mapping project whose subject is the G333 giant molecular cloud and which is known informally as the 'Delta Quadrant Survey' (DQS). In Section 6.1, Maria Cunningham outlines the DQS project. In Section 6.2, Paul Jones details work done on quantifying the turbulent structure in the G333 cloud via analysis of the spatial power spectrum and Nadia Lo presents the detection of an anomaly seen in the hyperfine structure of the HCN emission in Section 6.3. In Section 6.4, Shari Breen reports on a water maser follow-up study to the DQS. Finally, in Section 6.5, Indra Bains disseminates an offshoot of the DQS project, investigating regions of high mass star formation in the G333 cloud.

\section{First Results from the $\mathrm{H}_{2} \mathrm{O}$ Southern Galactic-Plane Survey}

Andrew Walsh In February/March 2008, the Mopra radiotelescope was used to begin a survey of the southern Galactic plane at $12 \mathrm{~mm}$. The $\mathbf{H}_{\mathbf{2}} \mathbf{O}$ Southern Galactic Plane Survey (HOPS, see Table 1; Walsh et al. 2008) aims to search for water masers at $22.235 \mathrm{GHz}, \mathrm{NH}_{3}(1,1),(2,2)$,

\footnotetext{
${ }^{3}$ See http : / / www . astro. uni-koeln. de/nanten2/.
}

$(3,3)$ and $(6,6), \mathrm{HC}_{3} \mathrm{~N}(3-2)$, many $\mathrm{CH}_{3} \mathrm{OH}$ thermal and maser lines as well as radio recombination lines ( $\mathrm{H} 69 \alpha$ and H62 $\alpha$ ). HOPS will cover 90 square degrees of the southern Galactic plane from $l=300-0-30$ and $|b|<0.5^{\circ}$, simultaneously covering 16 spectral windows between 19.5 and $27.5 \mathrm{GHz}$. This project will take approximately 3 years and over 2000 hours of telescope time to complete. So far, we have surveyed 31 square degrees from $l=300$ to 310 , 330 to 340 and -1 to 10 , detecting 199 water masers and about 500 ammonia cores. Most of these ammonia cores show emission in both the $(1,1)$ and $(2,2)$ inversion transitions, which can later be used to estimate the temperatures of the cores.

We detect strong, widespread emission towards the Galactic Centre (Figure 1) in ammonia $(1,1),(2,2),(3,3)$, $(6,6)$ and $(9,9)$, as well as cyanoacetylene, the H69 $\alpha$ radio recombination line, methanol and cyanodiacetylene. This clearly shows the central molecular zone is a unique region in our Galaxy, with widespread emission of high-energy transitions not seen in our HOPS observations of other parts of the Galaxy.

\section{The Central Molecular Zone of the Galaxy}

Michael Burton We are undertaking a molecular-line mapping survey of the Central Molecular Zone (or CMZ) of the Galaxy (see Table 1). The molecular environment in the inner few hundred parsecs of the Galaxy is a unique region for astrophysical investigations, containing approximately $10 \%$ of its molecular gas. It is a region rich in organic molecules, whose distribution is far more widespread than found in giant molecular clouds elsewhere in the Galaxy. The advent of the UNSW-MOPS spectrometer on Mopra, combined with on-the-fly mapping and the MMIC receiver, has made such an extensive study of the molecular content of the CMZ possible.

The CMZ extends approximately $3^{\circ}$ along the Galactic plane (though is not centred on it) and $0.25^{\circ}$ out of the plane (i.e. an extent of $\sim 450 \times 150 \mathrm{pc}$ ). It is prominent in molecular emission (in particular $\mathrm{CO}$ ) as well as in far-IR dust emission, indicating the presence of extensive star formation throughout it. It contains $\sim 10^{8} \mathrm{M}_{\odot}$ of molecular gas, about $10 \%$ of the total molecular content of the Galaxy. The IR emission similarly accounts for $\sim 10 \%$ of the Galaxy's IR luminosity. The CMZ is quite different in nature to molecular clouds found elsewhere in the Galaxy, such as in the 3-5-kpc Galactic Ring. Temperatures are in general significantly higher (typically $30-60 \mathrm{~K}$, though up to $200 \mathrm{~K}$, compared to $10-20 \mathrm{~K}$ in giant molecular clouds), densities exceed $10^{4} \mathrm{~cm}^{-3}$ throughout and turbulent velocities are high $\left(\sim 15-50 \mathrm{~km} \mathrm{~s}^{-1}\right.$ versus $\sim 5 \mathrm{~km} \mathrm{~s}^{-1}$ in GMCs). Elsewhere in the Galaxy such densities are only found in cloud cores, not extended over entire cloud complexes, but this must be necessitated by the need to withstand tidal shearing in the central regions. The CMZ is thus a very different environment to that in which star formation has been studied in either clouds nearby to the Sun or in the molecular clouds of the Galactic Ring. 
Table 1. Observing parameters of the millimetre-wavelength surveys in this publication

\begin{tabular}{lllrrr}
\hline $\begin{array}{l}\text { Survey } \\
(1)\end{array}$ & $\begin{array}{l}\text { Telescope } \\
(2)\end{array}$ & $\begin{array}{l}\text { Region } \\
(3)\end{array}$ & $\begin{array}{r}\text { Band }(\mathrm{mm}) \\
(4)\end{array}$ & $\begin{array}{r}\theta(\operatorname{arcsec}) \\
(5)\end{array}$ & $\begin{array}{c}\delta_{v}\left(\mathrm{~km} \mathrm{~s}^{-1}\right) \\
(6)\end{array}$ \\
\hline DQS & Mopra & $\sim 1^{\circ}$ square centred on $\sim \alpha_{\mathrm{J} 2000}=16: 21, \delta$ J2000 $=-50: 35$ & 3 & 36 & 0.1 \\
CMZ & Mopra & $l=-0^{\circ} .7$ to $1^{\circ} .8, b=-0^{\circ} .3$ to $0^{\circ} .2$ & 3 & 36 & 0.9 \\
HOPS & Mopra & $l=300^{\circ}$ to $30^{\circ},|b|=<0^{\circ} .5$ & 12 & 120 & 3.5 \\
MAGMA (LMC) & Mopra & $\alpha_{\mathrm{J} 2000}=06: 00$ to $04: 40, \delta_{\mathrm{J} 2000}=-71: 30$ to $-65: 00$ & 3 & 45 & 0.9 \\
MAGMA (SMC) & Mopra & $\alpha_{\mathrm{J} 2000}=01: 07$ to $00: 56, \delta_{\mathrm{J} 2000}=-72: 40$ to $-71: 40$ & 3 & 45 & 0.9 \\
NANTEN (LMC) & NANTEN & $\alpha_{\mathrm{J} 2000}=06: 00$ to $04: 30, \delta_{\mathrm{J} 2000}=-71: 00$ to $-65: 30$ & 3 & 156 & $0.1,0.6$ \\
NANTEN (SMC) & NANTEN & $\alpha_{\mathrm{J} 2000}=01: 30$ to $00: 40, \delta_{\mathrm{J} 2000}=-73: 50$ to $-71: 45$ & 3 & 156 & 0.1 \\
\hline
\end{tabular}

The columns are as follows: (1) survey name; (2) telescope used; (3) region covered in the survey; (4) observing band; (5) typical angular resolution; (6) typical channel width.

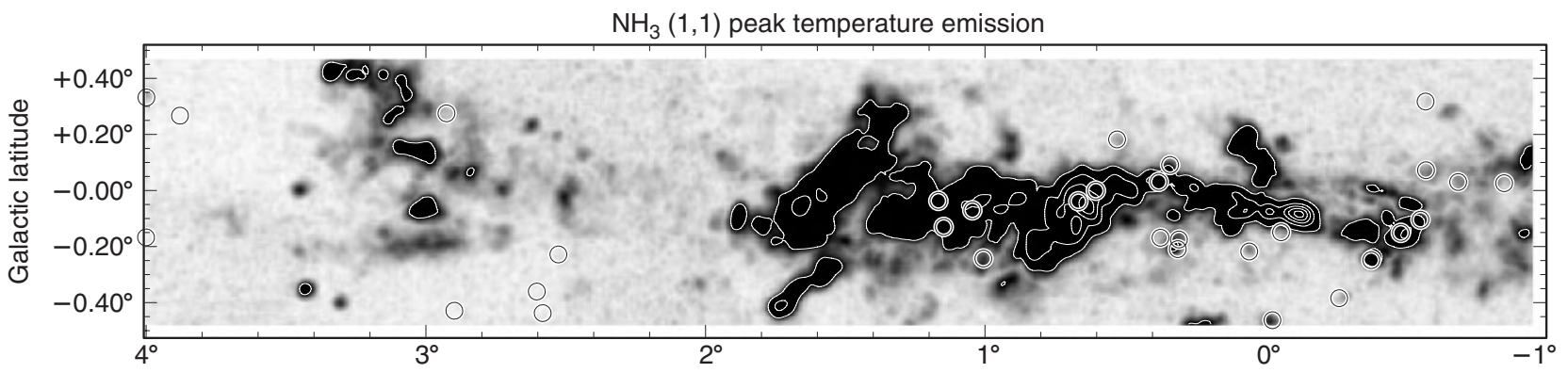

Figure 1 Ammonia $(1,1)$ peak temperature map for the region of HOPS covering the Central Molecular Zone. The circles indicate the positions of detected water masers. See Section 2 for more details.

Perhaps most surprisingly, the $\mathrm{CMZ}$ is rich in organic molecules and these appear to be widespread throughout it. Yet, while the prominent cloud of Sgr B2 in the CMZ is well studied (containing about $10 \%$ of the CMZ's mass, the largest column density along any sight line in the Galaxy, and virtually every exotic molecular species so far found in the ISM, or Interstellar Medium), the rest of the $\mathrm{CMZ}$ is little studied outside the $\mathrm{CO}$ lines. However, it is clear from $\mathrm{CH}_{3} \mathrm{OH}$ (methanol) measurements made at $834 \mathrm{MHz}$ with a beam of $40^{\prime}$ ( $\equiv \sim 100 \mathrm{pc}$ ), that the emission is extended even on this scale (Gottlieb et al. 1979). From HNCO (isocyanic acid) mapping at $110 \mathrm{GHz}$ with a 9' beam by Dahmen et al. (1997), made fortuitously at the same time as a $\mathrm{C}^{18} \mathrm{O}$ map of the $\mathrm{CMZ}$ (the line fell in the same bandpass), the organic species was seen to be extended on much the same scale as the far more abundant CO molecule. This was completely unexpected and still remains unexplained. While organic reservoirs are common in molecular clouds, they are confined to compact 'hot molecular cores' (HMCs, warm $\sim 100 \mathrm{~K}$, dense $\sim 10^{6-7} \mathrm{~cm}^{-3}$ knots inside GMCs), where massive star formation has recently started $\sim 10^{4-5}$ years ago (see, for instance, Purcell et al.'s 2006 Mopra survey). Hot molecular cores are of order $0.1 \mathrm{pc}$ in size, much smaller than the $\sim 10 \mathrm{pc}$ scales associated with GMC complexes, let alone the $\sim 100 \mathrm{pc}$ scale associated with the CMZ. The CMZ appears to be behaving like a hot molecular core extended over many parsecs. We know of no other region like this in the universe, though possibly it may represent the typical environment found in the central regions of rich, star forming galaxies (e.g. see Menten et al. 2004).

The morphology of the region is complex, characterised by expanding arcs, shells and filaments. Furthermore, the molecular line profiles are exceedingly broad across the entire CMZ, typically $15-30 \mathrm{~km} \mathrm{~s}^{-1}$ wide, but reaching up to $100 \mathrm{~km} \mathrm{~s}^{-1}$ in places. In addition, $\mathrm{SiO}$ emission is widespread, and this requires large-scale shocks or cloud-cloud collisions for its occurrence (Martin-Pintado et al. 1997). These may possibly be the result of the violent release of kinetic energy by some hundreds(?) of supernova explosions in the past $\sim 10^{4}$ years, for the CMZ region also contains extensive hot $\left(\sim 10^{8} \mathrm{~K}\right)$ plasma (Koyama et al. 1989) and $\gamma$-ray emission from the relatively short-lived ${ }^{26} \mathrm{Al}$ (von Ballmoos, Diehl \& Shoenfelder 1987).

We are now mapping the CMZ in an $8 \mathrm{GHz}$ band centred at $89 \mathrm{GHz}$ using the UNSW-MOPS, obtaining maps in 18 lines simultaneously. This is a project that will take three years to complete. A pilot project was undertaken in 2006, presenting maps of a $5^{\prime}$ region around Sgr B2 in 38 molecules and detecting $\sim 170$ lines (Jones et al. 2008). We then mapped a degree-sized region from Sgr B2 to Sgr A in 2007, and show a representative position-velocity plot in Figure 2, together with four sample integrated line maps in Figure 3. The instrument provides a resolution of $1 \mathrm{~km} \mathrm{~s}^{-1}$. With OTF mapping, a $5^{\prime} \times 5^{\prime}$ region can be mapped in $\sim 1$ hour of telescope time (inc. all overheads), yielding a sensitivity of $\mathrm{T}_{\mathrm{A}}^{*} \sim 0.1 \mathrm{~K}$. Data reduction is performed using the excellent Livedata and Gridzilla packages developed 


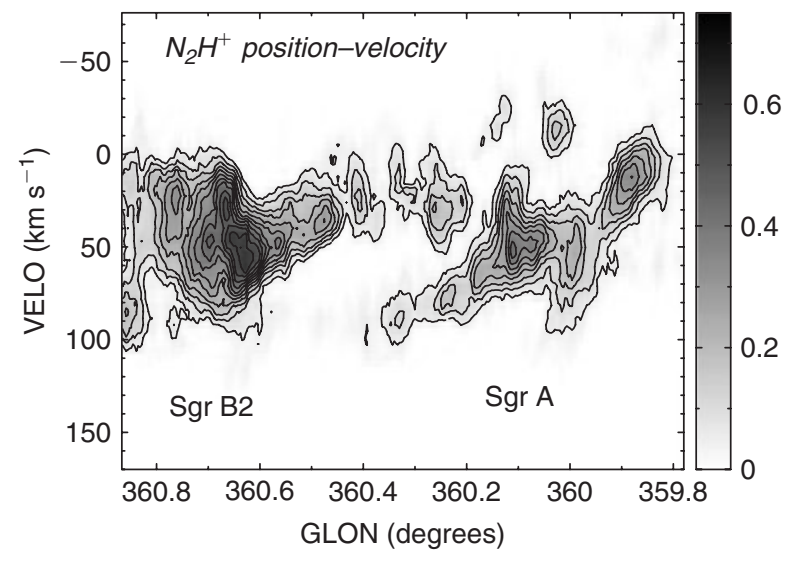

Figure 2 A representative position-velocity diagram from the CMZ data set, showing the dynamics in the $93.2 \mathrm{GHz} \mathrm{N} \mathrm{H}^{+}$ molecule. Despite generally being regarded as tracer of cold, dense molecular cores, line widths of up to $100 \mathrm{~km} \mathrm{~s}^{-1}$ are evident, indicating past violent activity across the CMZ, presumably related to shock waves driven by multiple supernova explosions. Our data demonstrate complex dynamical structures along a $\sim 100 \mathrm{pc}$ long molecular ridge extending from Sgr A to Sgr B2. See Section 3 for more details.
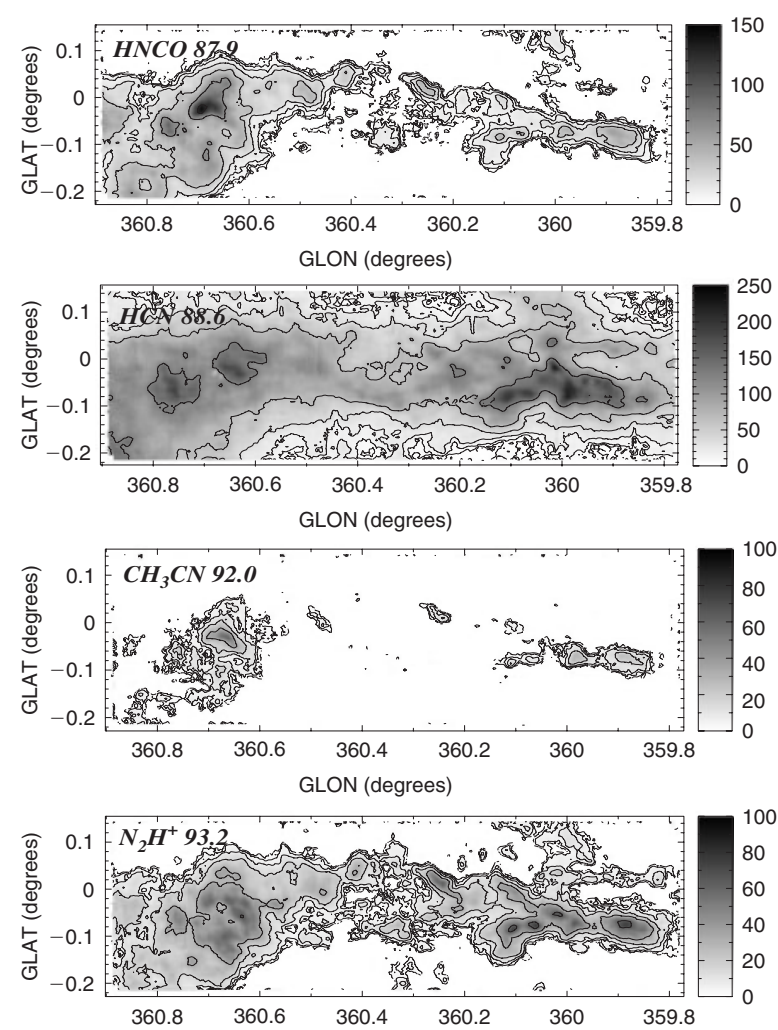

Figure 3 Examples of the integrated molecular line emission maps from the CMZ. From top to bottom: HNCO $(87.9 \mathrm{GHz})$; HCN (88.6 GHz); $\mathrm{CH}_{3} \mathrm{CN}(92.0 \mathrm{GHz})$ and $\mathrm{N}_{2} \mathrm{H}^{+}(93.2 \mathrm{GHz})$ across the inner $1^{\circ}$ of the CMZ. Sgr B2 is the prominent source to the left, and Sgr A to the right (the Galactic centre). While $\mathrm{HCN}$ is generally widespread in molecular clouds, the other three species are usually confined to dense cores within them, in contrast to their extended distribution here. $0.1^{\circ}$ represents $15 \mathrm{pc}$ at the $8.5 \mathrm{kpc}$ distance of the CMZ. See Section 3 for more details. by Mark Calabretta, with extensions added to handle the format of the MOPS data.

\section{Star Formation in the Magellanic Clouds}

Akiko Kawamura The galaxies that comprise the Magellanic System are the nearest neighbours to our own. The Large Magellanic Cloud (LMC) is nearly face-on, making it feasible to identify objects associated with the GMCs within it without suffering from contamination along the line-of-sight. Because of this observational advantage, observations covering almost the entire region of the Magellanic Clouds have been carried out in a wide range of wavelengths and have been bringing us important knowledge of the properties of the stars and ISM contained within.

A CO (1-0) survey of the Magellanic Clouds with a 4-m mm-wave telescope, NANTEN, has provided a rich sample of GMCs at a spatial resolution of $40 \mathrm{pc}$, well below the typical size of a GMC (Fukui et al. 2008, see Table 1). This survey complements previous surveys (Israel et al. 2003) by covering limited regions with high resolution and also plays a role as a guide for on-going higher resolution observations.

The sample of GMCs has been used to study how star formation is taking place by comparing the GMC distributions with optical signposts of star formation. This comparison clearly indicates that the distribution of the youngest clusters $(<10 \mathrm{Myr})$ and HiI regions are sharply peaked within $100 \mathrm{pc}$ of a GMC, while the older clusters show much weaker correlations. The GMCs are classified into three types according to their association with HII regions and clusters or associations as young as $10 \mathrm{Myr}$ (SWB 0 by Bica et al. 1996). It shows that $24 \%$ of the GMCs are without massive star formation (Type I), about a half of the GMCs are associated with small Hir region(s) only but without stellar clusters (Type II) and the rest (Type III) are most actively forming stars as shown by huge HII regions and young stellar clusters. A comparison of physical parameters among these three types indicates that size and mass tend to increase from Type I to Type III, and Type III GMCs have the largest size and mass among the three, while line width has a similar distribution among the three types. This typing can be interpreted as an indication of an evolutionary sequence from I to III and the lifetime of a GMC is estimated to be about $30 \mathrm{Myr}$ in total by comparing the lifetime of stellar clusters based on a steady-state assumption. The stage after Type III is perhaps a very violent dissipation of the GMCs due to UV photons and stellar winds from formed clusters, as seen most spectacularly in the region of 30 Dor.

To study the molecular cloud properties and their relation to star formation activities, we have been carrying out sub-mm observations with NANTEN2 and ASTE (Atacama Submillimeter Telescope Experiment). Their beam sizes correspond to 5 to $10 \mathrm{pc}$, enabling us to resolve the GMCs into dense clumps. We have mapped one of the most active star forming regions, N159, and also a GMC without massive star formation. The intensity ratio of $\mathrm{CO} J=3-2$ 
(ASTE data) and $J=4-3$ (NANTEN2 data) to CO $J=$ $1-0$ (Mopra data) is found to be higher where the $\mathrm{H} \alpha$ flux is higher, showing a good correlation of the temperature of the dense cores and star formation activities.

\section{$5 \mathrm{TeV}\left(\mathbf{1 0}^{12} \mathrm{eV}\right)$ Gamma-Ray Results and Links to mm-Surveys}

Gavin Rowell The past few years has seen the establishment of $\mathrm{TeV} \gamma$-ray astronomy, with the H.E.S.S. (High Energy Stereoscopic System) telescopes dramatically providing, for the first time, resolved images of individual objects (see Aharonian et al. 2007a, for latest results). Additional instruments such as MAGIC ${ }^{4}$ and VERITAS $^{5}$ are also providing exciting new results. TeV $\gamma$-rays provide a probe of extreme particle accelerators such as supernova remnants (SNRs), pulsars, massive stars and stellar clusters, and winds and jets associated with accretion processes.

High-energy $\gamma$ rays of energy of a few $\mathrm{GeV}$ and above can be produced in the collisions of cosmicray (CR) hadrons (protons, nuclei) with dense ambient matter such as in molecular clouds, or from the inverseCompton up-scattering of soft photon fields by CR electrons (Bremsstralung is an additional electronic process). It is the hadronic process which brings together the fields of $\mathrm{TeV} \gamma$-ray and $\mathrm{mm}$ astronomy. The key signature in establishing the hadronic nature of the particles is the spatial correlation between the $\gamma$-ray emission and molecular gas, with the expected $\mathrm{TeV}$ flux being proportional to the cloud mass and CR density (Aharonian 1991). Thus, mm-surveys of molecular clouds can provide the vital link in locating the accelerators of CR hadrons. The origin of these CRs is in fact the oldest unanswered question in astrophysics arising in modern times (see review by Hillas et al. 2006).

Two clear examples of this $\mathrm{TeV}$-molecular cloud correlation are the Galactic Centre Ridge (Aharonian et al. 2006) and the old-age SNR W 28 in which several TeV sources are seen (Aharonian et al. 2008, see Figure 4). The W $28 \mathrm{TeV}$ sources are well-matched to the molecular clouds traced by the NANTEN ${ }^{12} \mathrm{CO}(J=1-0)$ survey (Matsunaga et al. 2001). From this correlation, one can estimate the density of CRs in the vicinity of the clouds, providing new clues as to the location of CR accelerators.

Probing the molecular cloud density profile and dynamics also yields fundamental information on the diffusion properties of CRs and shocked environments in which the clouds are situated (Gabici, Aharonian \& Blasi 2007). In this context, Australia's Mopra telescope plays a key role due to its ability to probe many molecular lines (such as $\mathrm{NH}_{3}$ ) and hence trace very dense and high temperature gas. Mopra's abilities are complementary to the sub-mm performance of NANTEN2 in this context.

In 2008, a survey of the W $28 \mathrm{TeV}$ sources was carried out with Mopra and NANTEN2 and analysis of these

\footnotetext{
${ }^{4}$ See http : / / wwwmagic.mppmu.mpg.de/.

${ }^{5}$ See http: //veritas.sao.arizona.edu/.
}

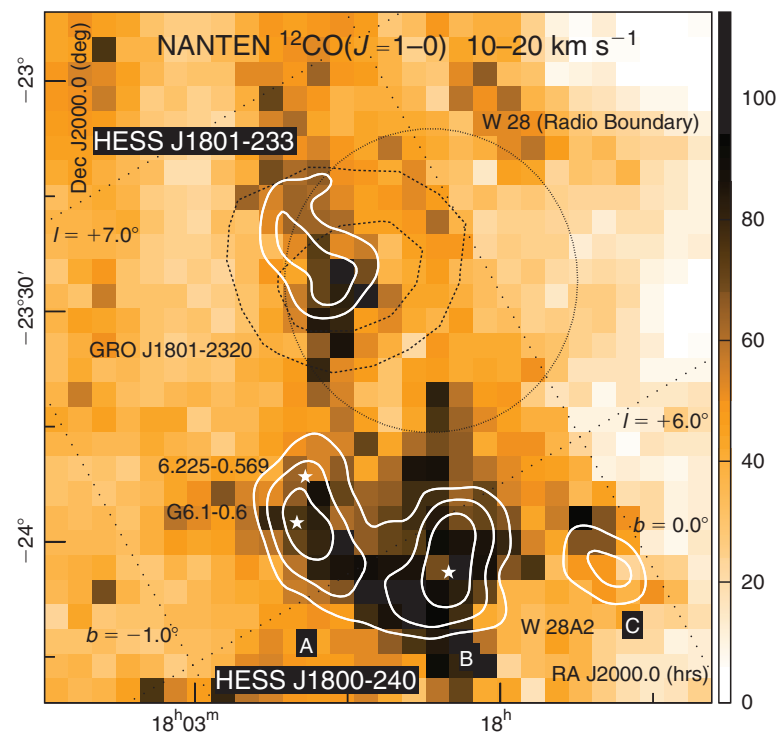

Figure 4 NANTEN integrated ${ }^{12} \mathrm{CO}(J=1-0)$ image of the W 28 region (from Aharonian et al. 2008). The linear colour scale is in $\mathrm{K} \mathrm{km} \mathrm{s}^{-1}$ for $V_{\mathrm{LSR}}=10$ to $20 \mathrm{~km} \mathrm{~s}^{-1}$, with HESS TeV $\gamma$-ray 4-, 5-, 6- $\sigma$ significance white contours. See Section 5 for more details.

data is underway. Looking to the future, detailed mmsurveys of bright young SNRs such as RX J1713.7-3946 (Aharonian et al. 2005a, 2007b) and RX J0852.0-4622 (Aharonian et al. 2005b, 2007c) where the shell-like $\mathrm{TeV}$ morphology is clear, are essential in establishing the hadronic nature of the parent accelerated particles in these objects. Such work will be a major step towards answering the question of Galactic CR origins.

\section{Science from the Mopra G333 Survey}

\subsection{Introducing the G333 'Delta Quadrant Survey'}

Maria Cunningham The Mopra mapping project informally known as the 'Delta Quadrant Survey' (DQS) is a multi-molecular line survey of the G333.6-0.2 GMC complex (see Table 1). It was motivated by the idea of gaining a comprehensive picture of massive star formation throughout an entire GMC, investigating its turbulent properties and how these might influence a future generation of star formation. The G333 complex spans a $1.2 \times 0.6$ degree region of the southern Galactic plane, and is around $3.6 \mathrm{kpc}$ distant from the Sun (Lockman et al. 1979). It is associated with the bright $\mathrm{H} \alpha$ emission region known as RCW106 (Rodgers, Campbell \& Whiteoak 1960). The complex shows many signs of active, massive star formation, including bright HII regions and IRAS sources (see e.g. Bains et al. 2006, and Section 6.5 of this paper), and forms part of the 3-5 kpc Galactic Ring (see e.g. Simon et al. 2001) of molecular emission. The region also forms part of the Spitzer GLIMPSE survey (see e.g. Benjamin, Whitney \& Churchwell 2008).

We have used the new broadband capabilities of the Mopra telescope detailed in Section 1 to map the full extent of the G333 GMC, in rotational transitions of 20 different molecules, with velocity resolutions in the range 
$0.1-0.2 \mathrm{~km} \mathrm{~s}^{-1}$ (see Bains et al. 2006; Lo et al. 2007; Wong et al. 2008, for more details). The DQS survey has produced a unique data set that is being used to answer observationally some of the key questions about the dynamical processes surrounding massive star formation (e.g. large-scale galactic flows and massive stellar winds; see Section 6.5) and their relative importance in regulating the star formation process. These dynamical processes drive the turbulent motions which are ubiquitous in GMCs.

Investigating and understanding the chemistry of this region is a necessary part of this project if the molecular line observations are to be correctly interpreted, and is an interesting goal in itself. In addition to the molecular line observations, we have observed continuum emission at sub-millimetre and centimetre wavelengths, and radio-recombination lines (see Section 6.5; Bains et al., in preparation). These observations are being combined with existing infrared and atomic hydrogen data to put together a comprehensive picture of massive star formation, and its relationship to the chemistry and dynamics of the entire cloud complex.

The first publications from this project (Bains et al. 2006; Wong et al. 2008) were based on use of the algorithms applied to decompose the emission of $\mathrm{CO}$ isotopomers from the G333 cloud into clumps that could be statistically analysed. In subsequent analyses of the cloud's statistical properties, we have used two techniques suitable for application to large data sets, both of which have yielded interesting results: the Spatial Power spectrum (SPS) and Principal Component analysis (PCA). We have investigated the SPS of a variety of molecules that trace different critical densities. Surprisingly, all molecules show a similar power law slope, suggesting a picture where turbulence is injected at large scales (at least tens of parsecs), and passes through to smaller scales, and higher densities, without significant dissipation (see Section 6.2). On the other hand, PCA analysis has shown that, despite the apparently uniform distribution of turbulent energy through the different molecular regions, different molecules are well differentiated at smaller scales. The PCA and has yielded some significant chemical correlations and anti-correlations, including an anti-correlation at small scales between the molecules $\mathrm{N}_{2} \mathrm{H}^{+}$and $\mathrm{HCO}^{+}$ (see Lo et al. 2009, for further details).

In addition to investigating the statistical properties of the large-scale GMC emission, various other interesting phenomena have been serendipitously discovered. Lo et al. (2007) reported on the discovery of a massive, cold dense core located within the $\mathrm{G} 333$ cloud, whose $\mathrm{SiO}(J=2-1)$ emission indicated the presence of an outflow from a future host of massive star formation. Another interesting discovery is presented in Section 6.3: the detection of anomalous hyperfine $\mathrm{HCN}$ emission.

Offshoots of the DQS project saw Breen et al. (2007) investigate the correlation between water maser emission and ${ }^{13} \mathrm{CO}$ and $1.2-\mathrm{mm}$ dust clump (the latter detailed in Mookerjea et al. 2004) properties; a further investigation based on the correlation between methanol maser
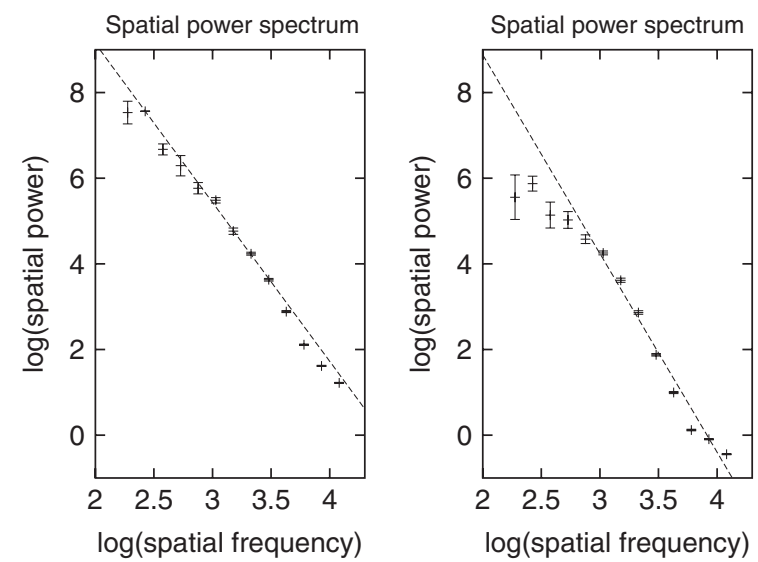

Figure 5 Spatial power spectra plots for ${ }^{13} \mathrm{CO}$ emission (left) corrected for optical depth, and CS emission (right). The good power law fit for ${ }^{13} \mathrm{CO}$ is typical, whereas the CS (from MOPS data) is unusual, showing a fall-off at small spatial frequencies due to a lack of large-scale structure. See Section 6.2 for more details.

emission and the above properties is presented in Section 6.4. Finally, to investigate the viability of massive stellar outflows as a source of the turbulence that can influence a future generation of star formation, Bains et al. (in preparation) have begun a multiwavelength observing campaign of the high-mass star-forming regions located within the cloud. Some of these regions show evidence for outflow in the Mopra DQS data, and these are being modelled and their driving sources investigated; preliminary results are presented in Section 6.5.

\subsection{Spatial Power Spectra of the G333 GMC}

Paul Jones The ISM is highly non-uniform, with distinct different phases. There are orders of magnitude differences in important parameters (e.g. density) even within the same phase. The molecular medium, in particular, is very clumpy. This can be described as a fractal structure. In the dynamic ISM, density fluctuations occur on a range of scales, with turbulent motions (and with some changes between phases). Large scale turbulent eddies drive smaller scale eddies in a cascade of energy, leading to the 'self-similar' structures.

One of the original motivations for the Mopra survey of the G333 region (Bains et al. 2006; Cunningham et al. 2007, see Section 6.1) was to study this turbulent structure (Jones et al. 2007), initially in ${ }^{13} \mathrm{CO}$ (Bains et al. 2006), but eventually in multiple 3-mm lines (Lo et al. 2009). We consider here the Spatial Power Spectrum (SPS) of the rotational emission lines of ${ }^{13} \mathrm{CO}, \mathrm{C}^{18} \mathrm{O}, \mathrm{CS}, \mathrm{HNC}$, $\mathrm{HCO}^{+}, \mathrm{HNC}$ and $\mathrm{N}_{2} \mathrm{H}^{+}$. The SPS is the power ( $P$ : square of the amplitude of the Fourier Transform of the image), as a function of spatial frequency $(k)$.

The main result is that all the molecules have similar statistical structure, even if there are differences in the detailed molecular distributions (Lo et al. 2007, 2009).

1. The spatial power spectra generally fit a good power law over the range of spatial scale from 1 to $20 \mathrm{pc}$, 
although some molecules show a deficit of large-scale power (see Figure 4).

2. The slope does not differ much for the different molecules (perhaps surprisingly) and is similar to the Kolmogoroff turbulence slope of $-11 / 3=-3.7$ for density fluctuations.

3. The optical depth affects the slope for ${ }^{13} \mathrm{CO}$, as correcting the intensities for optical depth, using the $\mathrm{C}^{18} \mathrm{O}$ as in Wong et al. (2008), boosts the small-scale structure.

4. The slope depends on the area within the G333 complex, with the central area having relatively more extended structure, and so steeper SPS slope.

5. The slope for individual spectral channels depends on the velocity, for the high $\mathrm{S} / \mathrm{N}{ }^{13} \mathrm{CO}$ data, where the line wings have slighly flatter SPS slopes than the line centre.

6. The slope depends on the velocity smoothing in the Velocity Channel Analysis (VCA; Lazarian \& Pogosyan 2000) of ${ }^{13} \mathrm{CO}$. The velocity integrated emission ('thick' slices) is not sensitive to the velocity fluctuations in the (2D spatial, 1D velocity) cube. The individual planes ('thin' slices), with velocity width smaller than the turbulent velocity, show structure due to velocity fluctuations. Hence the 'thick' and 'thin' slice slopes differ.

We are extending this analysis with complementary techniques: the delta variance (Bensch, Stutzki \& Ossenkopf 2001) to check the spatial power spectra, and the cross-power spectra and spatial coherency spectra (Stanimirovic et al. 2000), to study the correlation of the different molecules with each other.

\subsection{Detection of a HCN Hyperfine Anomaly in the G333 GMC}

Nadia Lo Following the distribution of molecular emission through a whole GMC can reveal results that may not be apparent in small maps made solely towards molecular emission peaks. One example of this is a hyperfine anomaly seen in the HCN emission from the G333 cloud, which becomes obvious away from the peak emission positions, where blending of the hyperfine components mask it.

$\mathrm{HCN}$ is a common molecule found in molecular clouds. Due to the nuclear quadrupole moment of ${ }^{14} \mathrm{~N}$, the $\mathrm{HCN}$ rotational transition displays hyperfine splitting. Under local thermal excitation, the relative hyperfine transition intensities of $J=1-0$ are 5:3:1 for $F=2-1, F=1-1$ and $F=0-1$, respectively. Shown in Figure 6 is an example $\mathrm{HCN}$ emission profile with a 'normal' hyperfine intensity ratio. From our HCN $J=1-0$ data cube, there are various regions which show hyperfine anomalies.

Shown in Figure 7 (top panel) is an example of the $\mathrm{HCN}$ hyperfine anomaly detected in the G333 molecular cloud, along with profiles of $\mathrm{HNC}$ (middle panel) and $\mathrm{N}_{2} \mathrm{H}^{+}$(bottom panel) emission taken at the same location. From the HCN profile it is clear that the $F=1-1$ transition is significantly weakened relative to that of the $F=2-1$. Indeed,

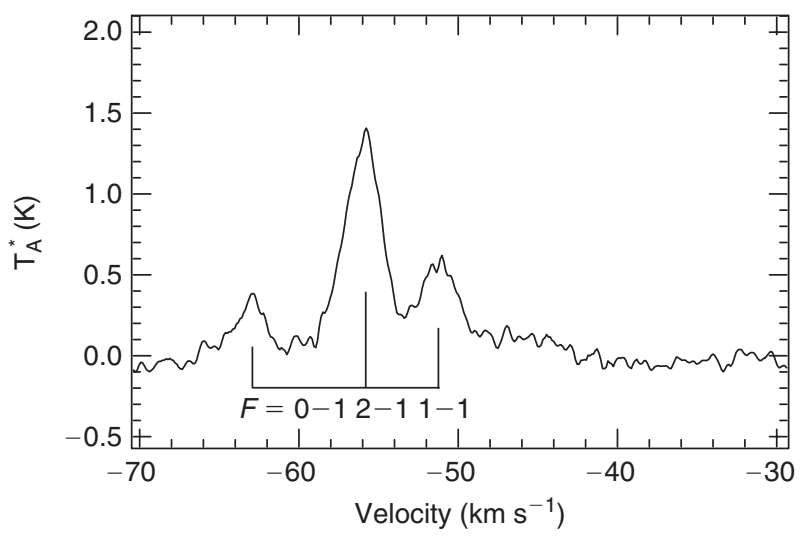

Figure 6 A 'normal' HCN profile from the G333 GMC, taken from $\alpha_{\mathrm{J} 2000}=16^{\mathrm{h}} 20^{\mathrm{m}} 06^{\mathrm{s}}, \delta_{\mathrm{J} 2000}=-50^{\mathrm{d}} 57^{\mathrm{m}} 12^{\mathrm{s}}$ and shown for comparison with the anomalous profile shown in Figure 7. See Section 6.3 for further details.

it is even weaker than the $F=0-1$ transition. The three hyperfine transitions were fitted with Gaussians using the CLASS $^{6}$ package; the fitted intensities are $0.77,0.13$ and $0.46 \mathrm{~K}$ for $F=2-1, F=1-1$ and $F=0-1$, respectively. The intensity ratios of the hyperfine components are 0.6 and 0.2 for $R_{02}=(\mathrm{F}=0-1) /(F=2-1)$ and $R_{12}=(F=1-$ $1) /(F=2-1)$, respectively. The expected LTE intensity ratios are $R_{02}=0.2$ and $R_{12}=0.6$; in this example, the observed $R_{12}$ is three times lower than the LTE ratio, and $R_{02}$ is three times higher. The hyperfine anomaly presented here is unlikely to be due to overlapping of multiple velocity components along the line-of-sight, as the geometrical isomer HNC emission profile shows there is only one velocity component peak at $-51 \mathrm{~km} \mathrm{~s}^{-1}$ (assuming $\mathrm{HCN}$ and $\mathrm{HNC}$ are tracing the same gas). Another molecule with hyperfine transitions is $\mathrm{N}_{2} \mathrm{H}^{+}$. Its emission profile does not show any hyperfine anomaly, further supporting the suggestion that this is unlikely to be caused by blending of velocity components.

Similar HCN hyperfine anomaly emission profiles to that shown in Figure 7 are present in various regions through out the G333 giant molecular cloud. This type of hyperfine anomaly has been observed toward warm clouds before (e.g. Gottlieb et al. 1975; Guilloteau et al. 1981). Guilloteau et al. (1981) concluded that in turbulent gas, the anomaly observed in the $J=1-0$ line is most likely due to overlap in velocity (blending) of components in the higherenergy $J=2-1$ transition. The hyperfine components in the higher energy transition are more closely spaced in frequency/velocity, leading to the transfer of populations between the hyperfine components in the $J=1$ energy level. However, a similar situation exists for the hyperfine transitions of the relevant transitions of $\mathrm{N}_{2} \mathrm{H}^{+}$, and which does not show any hyperfine anomaly.

Similar anomalies are observed in dark clouds, with narrow lines, where velocity overlap in the higher

\footnotetext{
${ }^{6}$ Continuum and Line Analysis Single-dish Software, part of GILDAS software package by IRAM. See http://www.iram. fr/IRAMFR/GILDAS/.
} 


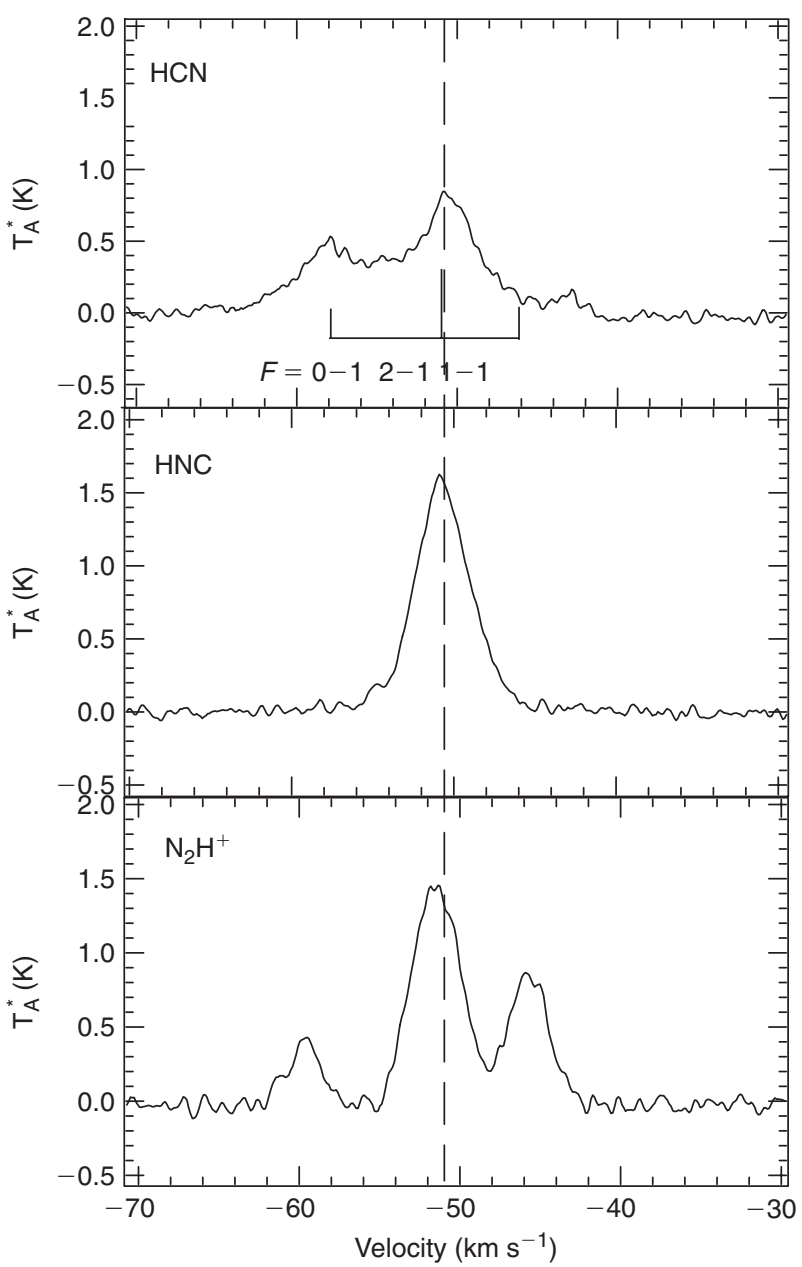

Figure 7 An example of the HCN hyperfine anomaly observed in the G333 GMC, shown with $\mathrm{HNC}$ and $\mathrm{N}_{2} \mathrm{H}^{+}$profiles taken at the same position: $\alpha_{\mathrm{J} 2000}=16^{\mathrm{h}} 21^{\mathrm{m}} 38^{\mathrm{s}}, \delta_{\mathrm{J} 2000}=-50^{\mathrm{h}} 23^{\mathrm{m}} 32^{\mathrm{s}}$. See Section 6.3 for further details.

transition cannot provide an explanation. Modeling by Gonzalez-Alfonso \& Cernicharo (1993) showed that hyperfine anomalies can also be explained by the presence of infall and/or outflow, if combined with a complex cloud density structure. There is evidence that $\mathrm{N}_{2} \mathrm{H}^{+}$is tracing particularly cool gas, and is not involved in outflow, and so the fact that $\mathrm{N}_{2} \mathrm{H}^{+}$does not show anomalies towards the same positions where $\mathrm{HCN}$ does, is consistent with outflow, traced by $\mathrm{HCN}$, being the cause. We comment that in the G333 region, the hyperfine anomalies are most pronounced towards regions associated with outflow, and also possibly infall (see Section 6.5). We also comment that there is no strong relationship between the peak intensity of the $J=1-0, F=2-1$ component (the strongest) and the appearance of the anomaly (cf. Figures 6 and 7), nor is there any obvious relationship between the presence of the anomaly and line width: different positions with very similar line widths in the $F=2-1$ transition may show the anomaly or not. However, we stress that this is work in progress, and we have not yet made a rigorous statistical analysis of the relationship between the relative strengths of the hyperfine components and absolute intensity or line width. We are currently working on making both physical models for these sources and on modelling the HCN line emission. For the latter, observations of the HCN 2-1 transition are needed. The mixture of normal and anomalous profiles throughout the region will be a significant aid in this work.

\subsection{Masers in the G333 GMC}

Shari Breen Masing emission from water, methanol and hydroxyl molecules is known to trace star formation, although a satisfactory evolutionary classification scheme based on these diagnostic probes is yet to be formulated. To further our understanding of this matter, the properties of the ${ }^{13} \mathrm{CO}$ clumps observed by Bains et al. (2006; see Section 6.1) and the 1.2-mm dust clumps observed by Mookerjea et al. (2004) in the regions of the G333 giant molecular cloud with associated water masers, were investigated by Breen et al. (2007). Comparison of the locations of the ${ }^{13} \mathrm{CO}$ and 1.2-mm dust clumps with the water masers showed that every water maser within the region was associated with both a molecular gas and dust emission peak (or clump). Breen et al. (2007) found that there was a strong increase in likelihood of water maser presence with increasing values of all tested properties: clump radius, mass, column density and brightness, for both ${ }^{13} \mathrm{CO}$ and $1.2 \mathrm{~mm}$ dust clumps, and presented models that are able to predict the probability of water maser presence where suitable $1.2 \mathrm{~mm}$ dust or ${ }^{13} \mathrm{CO}$ clump data is available.

We have repeated the analysis carried out by Breen et al. (2007), this time investigating the properties of the ${ }^{13} \mathrm{CO}$ and $1.2 \mathrm{~mm}$ dust clumps associated with $6.7 \mathrm{GHz}$ methanol masers (Ellingsen 2005). Comparison of the locations of the methanol masers with the molecular gas and dust data showed that all 5 of the methanol masers that lie within the regions surveyed by Bains et al. (2006) and Mookerjea et al. (2004) are associated with both molecular gas and dust emission peaks. In the regions completely searched for both methanol masers and ${ }^{13} \mathrm{CO}$ clumps there are $56{ }^{13} \mathrm{CO}$ clumps with no associated methanol masers and $5{ }^{13} \mathrm{CO}$ clumps with associated methanol masers. In the case of the 1.2-mm dust clumps there are 90 clumps with no associated methanol masers and 5 with associated methanol masers.

A Binomial generalized linear model (GLM) (McCullagh \& Nelder 1989) was fitted to the maser presence/absence data. A GLM predicts the probability of finding a methanol maser in a given 1.2-mm dust or ${ }^{13} \mathrm{CO}$ clump, using the respective clump properties as predictors. In the case of the ${ }^{13} \mathrm{CO}$ clumps, the properties considered were the integrated flux density of the clump peaks $\left(10 \mathrm{~K} \mathrm{~km} \mathrm{~s}^{-1}\right)$, peak flux density in terms of antenna temperature $(\mathrm{K})$, clump radius $(\mathrm{pc}),{ }^{13} \mathrm{CO}$ column density $\left(10^{16} \mathrm{~cm}^{-2}\right)$ and the total LTE molecular mass calculated from the ${ }^{13} \mathrm{CO}$ data. In the case of the $1.2 \mathrm{~mm}$ dust clump analysis the properties $F_{\text {peak }}\left(\mathrm{mJy}\right.$ beam $\left.^{-1}\right)$, radius $(\mathrm{pc})$, $F_{v}(\mathrm{Jy})$, mass $\left(\mathrm{M}_{\odot}\right)$ and $n_{\mathrm{H}_{2}}\left(10^{4} \mathrm{~cm}^{-3}\right)$ were investigated as predictors. 
The significance of all individual clump properties were tested by fitting all possible single term models and compared by analysis of deviance to the null model consisting only of an intercept. There is an increased likelihood of methanol maser presence associated with ${ }^{13} \mathrm{CO}$ clumps with increasing values of integrated flux density and column density. In the case of the 1.2-mm dust clumps, we find that there is an increased likelihood of methanol maser presence associated with increasing values of column density, radius and mass.

Box plots were created for each of the 1.2-mm dust clump properties and are shown in Figure 8. The solid horizontal line in each of these plots represents the median of the data. The box represents the 25 th to the 75 th percentile, while the vertical dashed line from the top of the box represents data from the 75 th percentile to the maximum value and the vertical line from the bottom of the box represents data from the 25 th percentile to the minimum value. Extreme values of clump properties are classified as outliers, and represented by dots, if their range from the 25 th or 75 th percentile (in the case of the minimum and maximum values of clump properties, respectively) is greater than 1.5 times that of the interquartile range.

Stepwise model selection based on the Akaike Information Criteria (Burnham \& Anderson 2002) was used to select the simplest model with the greatest predictive powers. In the case of the ${ }^{13} \mathrm{CO}$ analysis the estimated regression relation is

$$
\log \frac{p_{\mathrm{i}}}{1-p_{\mathrm{i}}}=-5.56+1.47 x_{\text {integ }}-1.53 x_{\text {peak }},
$$

where $p_{\mathrm{i}}$ is the probability of methanol maser presence in the $i$ th clump, $x_{\text {integ }}$ represents the integrated flux density of the clump peak $\left(10 \mathrm{~K} \mathrm{~km} \mathrm{~s}^{-1}\right), x_{\text {peak }}$ represents the clump peak in terms of antenna temperature $(\mathrm{K})$.

The estimated regression relation for methanol maser presence associated with $1.2-\mathrm{mm}$ dust clumps is

$$
\begin{aligned}
\log \left(\frac{p_{\mathrm{i}}}{1-p_{\mathrm{i}}}\right)= & -94.55+0.00151 x_{F \text { peak }}+78.3 x_{\text {radius }} \\
& -2.24 x_{F_{v}}-0.000836 x_{\text {mass }} \\
& +10.7 x_{\text {density }}
\end{aligned}
$$

where $p_{\mathrm{i}}$ is the probability of methanol maser presence in the $i$ th clump, $x_{F \text { peak }}$ is the peak flux density per beam of the clump $\left(\mathrm{mJy}\right.$ beam $\left.^{-1}\right), x_{\text {radius }}$ is the clump radius (pc), $x_{F_{v}}$ is the integrated flux density (Jy), $x_{\text {mass }}$ is clump mass in units of solar masses and $x_{\text {density }}$ is column density $\left(10^{4} \mathrm{~cm}^{-3}\right)$.

These models have encouraging misclassification rates, although could benefit from some refinements by testing a larger sample. In particular, the ${ }^{13} \mathrm{CO}$ model for methanol maser presence predicts that nearly all of the ${ }^{13} \mathrm{CO}$ clumps have a very low probability of methanol maser presence although it does predict that the clumps with associated methanol masers have a slightly higher probability of methanol maser presence than those clumps without associated methanol masers. The 1.2-mm dust clump model
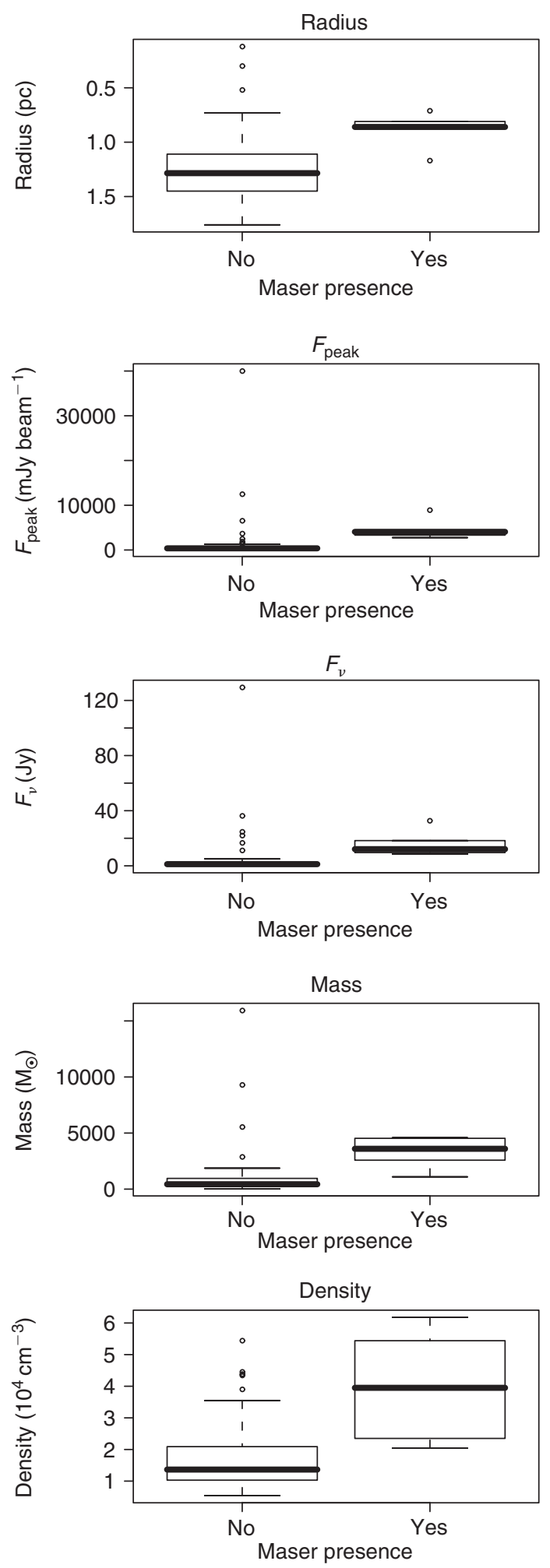

Figure 8 Box plots of the 1.2-mm dust clump properties split into the categories of yes and no, referring to methanol maser presence and absence, respectively. See Section 6.4 for more details.

has better predictive properties. If we set the probability threshold to 0.5 (i.e. those clumps with a value greater than 0.5 suggests the presence of a methanol maser, while a lower value suggests no methanol maser), the model correctly predicts 89 of the 90 clumps within the region with 


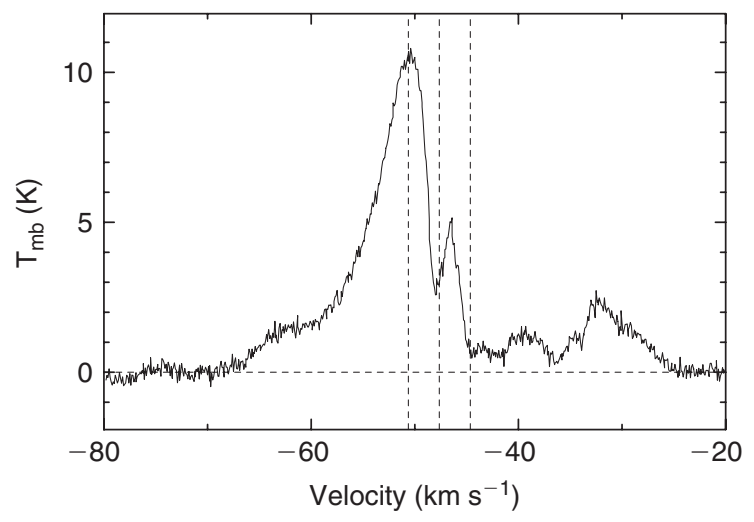

Figure 9 Continuum-subtracted velocity profile of ${ }^{12} \mathrm{CO}$ emission from the high-mass star-forming region G333.6-0.2. Broad outflow wings and deep absorption features are apparent. See Section 6.5 for more details.

no associated methanol masers and 3 of the 5 clumps with associated methanol masers.

We find that the ${ }^{13} \mathrm{CO}$ and $1.2-\mathrm{mm}$ dust clumps associated with the methanol masers are generally less extreme than those associated with the water masers. The multitude of data becoming available for the G333.2-0.6 GMC, ranging from complete searches of water, methanol and main-line $\mathrm{OH}$ masers to many molecular line transitions as well as 1.2-mm dust continuum emission, provides us with a unique opportunity to continue our investigation of the relative evolutionary stages that different maser species may be tracing.

\subsection{High-Mass Star-Forming Regions in the G333 GMC}

Indra Bains Some of the areas of brightest molecular emission in the Mopra maps of the G333 cloud (Bains et al. 2006; Wong et al. 2008; Lo et al. 2009) are associated with high-mass star-forming regions (HMSFRs) and show structures in the velocity profiles that are characteristic of outflow and infall (we show the most extreme example in Figure 7). We (Bains et al., in preparation) are currently modelling the line profiles to investigate, amongst other things, the amount of energy returned to the ISM by the outflows. This feedback from massive star formation is one of the postulated sources of the interstellar turbulence that is thought able to trigger a future generation of star formation (e.g. Mac Low \& Klessen 2004).

To characterise the HMSFRs themselves and locate the possible driving sources of the outflows, we have made multi-frequency radio continuum observations of the emission from the HII regions within them, using the Australia Telescope Compact Array (ATCA). Maps of the radio continuum emission from one of the HMSFRs, associated with the infrared source IRAS 16164-5046, reveal a compact radio source that is optically thick at least up to $22 \mathrm{GHz}$. The optically thick spectral indices of this source are $\alpha_{1384}^{2368}=3.0, \alpha_{2368}^{4800}=1.2$ and $\alpha_{4800}^{8640}=0.6$, which are consistent with those expected from a thermal jet (Reynolds 1986). At $22 \mathrm{GHz}$, the source has a deconvolved size of $0.03 \mathrm{pc}$. Assuming $\tau_{22 \mathrm{GHz}} \sim 1$ and using a
$3.6 \mathrm{kpc}$ distance to the GMC (Lockman et al. 1979), the number of ionizing photons is consistent with 1 ZAMS O 6.5 star (Panagia 1973, this is a lower limit as the spectrum appears to be turning at this frequency), with an emission measure of $1.4 \times 10^{9} \mathrm{pc} \mathrm{cm}^{-6}$ and a mass of $0.1 \mathrm{M}_{\odot}$. These properties are all consistent with those of an ultracompact HII region. Assuming a dust opacity index of 2 and a gas-to-dust ratio of 100 , the optically thin 3-mm $(89 \mathrm{GHz})$ dust mass of this source is $2.1 \times 10^{3} \mathrm{M}_{\odot}$ (for an assumed $\left.T_{\mathrm{d}}=40 \mathrm{~K}\right)$, or $4.4 \times 10^{3} \mathrm{M}_{\odot}\left(\right.$ for $\left.T_{\mathrm{d}}=20 \mathrm{~K}\right)$. Because this object also shows a molecular line profile consistent with both outflow and infall, we surmise that the $\mathrm{O} 6.5$ star is still accreting material and powering the large-scale molecular ouflow.

\section{Summary}

In this publication, we have presented work which has arisen from various recent or ongoing large-scale mapping projects with the Mopra and/or NANTEN2 millimetrewavelength telescopes. This work has been facilitated by recent upgrades to the instruments' hardware. It is due to the success demonstrated by these projects that a new wave of pilot studies for a number of multi-collaborative, international and larger-scale mapping projects has recently been instigated. These include the MALT (Millimetre Astronomers Large-area multi-Transition) 90-GHz Mopra survey, a 3+7-mm Mopra survey and a 7-mm ATCA survey. These projects are still in the proposal stage and if successful, their results will appear in future publications.

\section{Acknowledgments}

We thank Swinburne University of Technology for hosting the workshop and providing refreshments. IB acknowledges research support from Swinburne University. We thank the referee for helpful comments.

\section{References}

Aharonian, F., 1991, Ap\&SS, 180, 305

Aharonian, F. et al., 2005a, Natur, 432, 75

Aharonian, F. et al., 2005b, A\&A, 437, L7

Aharonian, F. et al., 2006, Natur, 439, 695

Aharonian, F. et al., 2007a, H.E.S.S. Collaboration Contribution to Proc. 30th ICRC (astro-ph/0710.4057)

Aharonian, F. et al., 2007b, A\&A, 464, 235

Aharonian, F. et al., 2007c, ApJ, 661, 236

Aharonian, F. et al., 2008, A\&A, 481, 401

Bains, I. et al., 2006, MNRAS, 367, 1609

Benjamin, R. A., Whitney, B. \& Churchwell, E., 2008, ASPC, 381 , 109

Bensch, F., Stutzki, J. \& Ossenkopf, V., 2001, A\&A, 366, 636

Bica, E., Claria, J. J., Dottori, H., Santos, J. F. C., Jr. \& Piatti, A. E., 1996, ApJS, 102, 57

Breen, S. L. et al., 2007, MNRAS, 377, 491

Burnham, K. P. \& Anderson, D. R., 2002, Model Selection and Multimodel Inference, A Practical Information - Theoretic Approach (2nd ed.; New York: Springer)

Cunningham, M. R., Bains, I., Lo, N., Wong, T., Burton, M. G. \& Jones, P. A., 2007, IAUS, 237, 404

Dahmen, G. et al., 1997, A\&AS, 126, 197

Ellingsen, S. P., 2005, MNRAS, 359, 1498

Fukui, Y. et al., 2008, ApJS, 178, 56 
Gabici, S., Aharonian, F. A. \& Blasi, P., 2007, Ap\&SS, 309, 365

Gonzalez-Alfonso, E. \& Cernicharo, J., 1993, A\&A, 279, 506

Gottlieb, C. A., Lada, C. J., Gottlieb, E. W., Lilley, A. E. \& Litvak, M. M., 1975, ApJ, 202, 655

Gottlieb, C., Ball, J., Gottlieb, W. \& Dickinson, D., 1979, ApJ, 227, 422

Guilloteau, S. \& Baudry, A., 1981, A\&A, 97, 213

Hillas, A. M., 2006, in Proc. Cosmology, Galaxy Formation and Astroparticle Physics on the Pathway to the SKA, Eds. Klöckner, H.-R., Rawlings, S., Jarvis, M. \& Taylor, A. (Oxford, University of Oxford), 9 (astro-ph/0607109)

Israel, F. P. et al., 2003, A\&A, 406, 817

Jones, P. A., Cunningham, M. R., Bains, I., Muller, E., Wong, T. \& Burton, M. G., 2007, IAUS, 237, 429

Jones, P. et al., 2008, MNRAS, 386, 117

Koyama, K., Awaki, H., Kunieda, H., Takano, S. \& Tawara, Y., 1989 Natur, 339, 603

Lazarian, A. \& Pogosyan, D., 2000, ApJ, 537, 720

Lo, N., Cunningham, M., Bains, I., Burton, M. G. \& Garay, G., 2007, MNRAS, 381, L30

Lo, N. et al., 2009, MNRAS, 395, 1021L

Lockman, F. J., 1979, ApJ, 232, 761

Mac Low, M.-M. \& Klessen, R. S., 2004, RvMP, 76, 125

Martin-Pintado, J., de Vicente, P., Fuente, A. \& Planeasa, P., 1997, ApJ, 482, L45
Matsunaga, K., Mizuno, N., Moriguchi, Y., Onishi, T., Mizuno, A. \& Fukui, Y., 2001, PASJ, 53, 1003

McCullagh, P. \& Nelder, J. A., 1989, Generalized linear models (London: Chapman and Hall)

Menten, K., 2004, in The Dense ISM in Galaxies, Proc. 4th CologneBonn-Zermatt Symp., Eds. Pfalzner, S., Kramer, C., Staubmeier, C. \& Heithausen, A. (Berlin, Heidelberg: Springer), 69

Mookerjea, B., Kramer, C., Nielbock, M. \& Nyman, L., 2004, A\&A, 426, 119

Ott, J. et al., 2008, PASA, 25, 129

Panagia, N., 1973, AJ, 78, 929

Purcell, C. et al., 2006, MNRAS, 367, 553

Reynolds, S. P., 1986, ApJ, 304, 713

Rodgers, A. W., Campbell, C. T. \& Whiteoak, J. B., 1960, MNRAS, 121,103

Simon, R., Jackson, J. M., Clemens, D. P., Bania, T. M. \& Heyer, M. H., 2001, ApJ, 551, 747

Stanimirovic, S., Staveley-Smith, L., van der Hulst, J. M., Bontekoe, T. R., Kester, D. J. M. \& Jones, P. A., 2000, MNRAS, 315, 791

von Ballmoos, P., Diehl, R. \& Schoenfelder, V., 1987, ApJ, 318,654

Walsh, A. J., Lo, N., Burton, M. G., White, G. L., Purcell, C. R., Longmore, S. N., Phillips, C. J. \& Brooks, K. J., 2008, PASA, 25105

Wong, T. et al., 2008, MNRAS, 386, 1069 